\title{
Management of Hydrofluoric Acid Burns in the Emergency Department
}

\author{
Jason Lippert ${ }^{1}$, Bobby Desai ${ }^{1}$, Michael Falgiani ${ }^{1}$, Trilok Stead $^{2}$, Latha Ganti ${ }^{3,4}$, 5 \\ 1. Emergency Medicine, Ocala Regional Medical Center, University of Central Florida College of Medicine, Ocala, USA \\ 2. Emergency Medicine, Trinity Preparatory School, Winter Park, USA 3. Emergency Medicine, Envision Physician \\ Services, Orlando, USA 4. Emergency Medicine, University of Central Florida College of Medicine/Hospital Corporation \\ of America Graduate Medical Education Consortium of Greater Orlando, Orlando, USA 5. Emergency Medicine, Polk \\ County Fire Rescue, Bartow, USA
}

Corresponding author: Latha Ganti, latha.ganti@ucf.edu

\begin{abstract}
Hydrofluoric acid burns are uncommon but unique among chemical burns in that they can cause visually mild burns with significant deep tissue injury and systemic toxicity through multiple mechanisms. We present the case of a patient who presented with bilateral hydrofluoric acid burns to his hands from aluminum brightener. The patient had been using an aluminum brightener with a hydrofluoric acid concentration of $10 \%$ for several months at work. On emergency department presentation, the patient endured significant tenderness to his hands and fingers. The patient suffered no serious complications, had no concerning lab or electrocardiographic findings, and was treated symptomatically with calcium gluconate gel. He was discharged home after successful symptom resolution with proper return precautions and instructions on how to safely use hydrofluoric acid containing products. Although not a very common cause of burns, acute care of these burns requires specific knowledge which is imperative for emergency personnel.
\end{abstract}

Received 02/24/2020 Review began 02/28/2020 Review ended 02/28/2020 Published 03/01/2020

\section{(c) Copyright 2020}

Lippert et al. This is an open access article distributed under the terms of the Creative Commons Attribution License CC-BY 4.0., which permits unrestricted use, distribution, and reproduction in any medium, provided the original author and source are credited.
Categories: Dermatology, Emergency Medicine Keywords: hydrofluoric acid, calcium gluconate

\section{Introduction}

Hydrofluoric acid (HFA) is an inorganic acid commonly used in both domestic and industrial settings for cleaning agents, fertilizer production, and many other uses [1,2]. In the household, HFA can be found in products such as silverware cleaner and herbicides. In the laboratory, HFA is used to remove rust, etch glass, and clean brass and crystal. It is also used in the production of semiconductor chips and gasoline [3]. In the dental industry, HFA is used as an etchant agent for ceramic materials [2].

Although a weak acid, at concentrations $>20 \%$, HFA can cause life-threatening caustic injury. In addition to corrosive burns, HFA is lipophilic, leading to deeper burns with liquefaction necrosis. On a systemic level, HFA releases fluoride ions that bind to calcium and magnesium, leading to hypocalcemia and hypomagnesemia, respectively. As a result, HFA can lead to significant systemic effects such as hypotension, cardiac arrhythmias, and cardiac failure [1].

Classically, physical examination demonstrates pain out of proportion to exam. Patients may have a delayed onset of symptoms of up to 24 hours, depending on the concentration. Patients can develop necrosis, ulceration, or gray areas at sites of exposure.

\section{Case Presentation}

A 37-year-old male presented to our emergency department (ED) with bilateral, burning hand pain, specifically under his nail beds and at his fingertips. The patient was exposed to aluminum brightener while at work, involving both of his hands. The patient did not use gloves and had been working without any issues for several months before this presentation. The product had an HFA concentration of $10 \%$.

The patient was hemodynamically stable on presentation, including initial vitals of heart rate of 86 beats per minute, respiratory rate of 18 breaths per minute, and a blood pressure of 138/78 mmHg. On physical examination, the patient had bilateral tenderness to both hands, but did not demonstrate any external findings such as erythema, necrosis, ulcerations, or discoloration. No significant neurological or vascular deficiencies were found for either extremity. The remainder of the physical examination was unremarkable.

The patient's medical workup included an ECG, basic metabolic panel, complete blood count, and magnesium and calcium concentrations. The local poison control center was contacted, who agreed with the workup and recommended applying calcium gluconate $(\mathrm{CaG})$ gel to the affected areas until the patient had complete relief of his pain. 


\section{Cureus}

ECG was negative for significant findings, including interval prolongations and arrhythmias. All lab results were within normal limits, including calcium and magnesium concentrations. The patient was treated with CaG gel, made in the ED by taking one gram of CaG and mixing it with standard lubricating gel in the ED. He received a total of three treatments of this gel before he had complete resolution of his pain.

Per the poison control center, once the patient was asymptomatic, with a normal ECG and lab values, he could be discharged home. The patient was given instructions concerning return precautions and instructions on how to safely use HFA products, including the use of appropriate gloves. He was then discharged home.

\section{Discussion}

HFA burns are uncommon but unique among chemical burns in that they can cause visually mild burns with significant deep tissue injury and systemic toxicity through a variety of mechanisms. The majority of medical literature discussing these burns is derived from case reports, small case series, animal studies, and anecdotal evidence [1].

HFA has multiple mechanisms of injury (Table 1), leading to the wide range of presentations. At high enough concentrations, HFA releases hydrogen ions, leading to visible tissue destruction, similar to other acid burns $[1,4]$. However, the majority of HFA burns are low concentration, and do not present with immediate corrosive destruction, and also may not have immediate pain [1]. At lower concentrations, HFA is lipophilic, leading to deep tissue injury due to to liquefaction necrosis. Once the fluoride ions dissociate they are easily absorbed into the bloodstream. These ions may then bind to calcium and magnesium cations, leading to decreased serum concentrations and their associated systemic effects [1,2]. Hypocalcemia is associated with perioral numbness, paresthesias, muscle cramps, focal or generalized weakness, and peripheral neuromuscular irritability that manifests as tetany. Depletion of calcium causes inhibition of the sodium potassium ATPase pump which results in cellular membrane permeability of potassium leading to hyperkalemia [5].

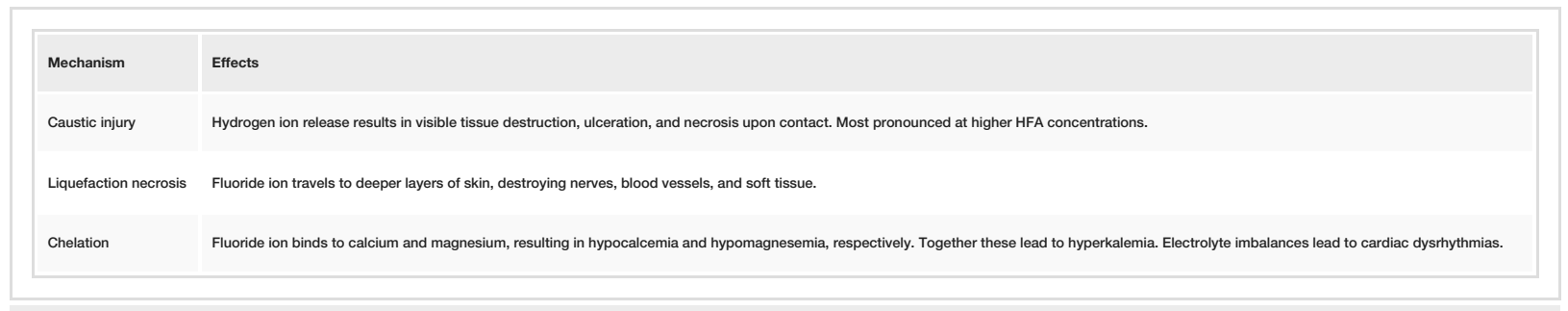

TABLE 1: Mechanisms of action of hydrofluoric acid (HFA)

Hypomagnesemia is associated with neuromuscular excitability and cardiac dysrhythmias. ECG abnormalities that can occur include prolonged QTc (from hypocalcemia), QRS widening (from hyperkalemia), polymorphic ventricular tachycardia (from hypomagnesemia), and T-wave elevation [5]. These cardiac dysrhythmias put HFA-exposed patients at risk for sudden cardiac death [2].

Skin symptoms are directly related to the acid concentration. Concentrations greater than $50 \%$ can cause immediate severe throbbing pain, with whitish discoloration of the skin and vesicles surrounded by an erythematous flare.These eventually transform into blisters containing necrotic tissue [2]. Should the burn affect the hands or fingers, reduced motor activity, decreased sensitivity, and even ischemia may result from arterial vasospasm [2]. More dilute solutions (concentrations ranging from $20 \%$ to $50 \%$ ) cause significant injury as well; however, symptoms may be delayed up to eight hours [4]. Lower concentrations (below $20 \%$ HFA) produce symptoms that tend to be delayed from 12 hours to up to several days after exposure, with no immediate pain [2]. The characteristic "pain out of proportion to exam" that these burns are known for is due to local neural hypocalcemia causing neuronal misfiring resulting in neuropathic pain.

Systemic toxicity is associated with any burns $>50 \%$ concentration, and exposure of $>5 \%$ of total body surface area regardless of concentration [6,7]. In addition to the electrolyte imbalances and cardiac dysrhythmias noted above, an excess of fluoride ions may lead to renal dysfunction, insufficiency, and renal cortical necrosis [2].

The initial treatment of HFA burns starts with a quick airway-breathing-circulation assessment, removal of contaminated clothing, and double bagging these contaminated articles to prevent secondary exposure (Figure 1). 


\section{Cureus}

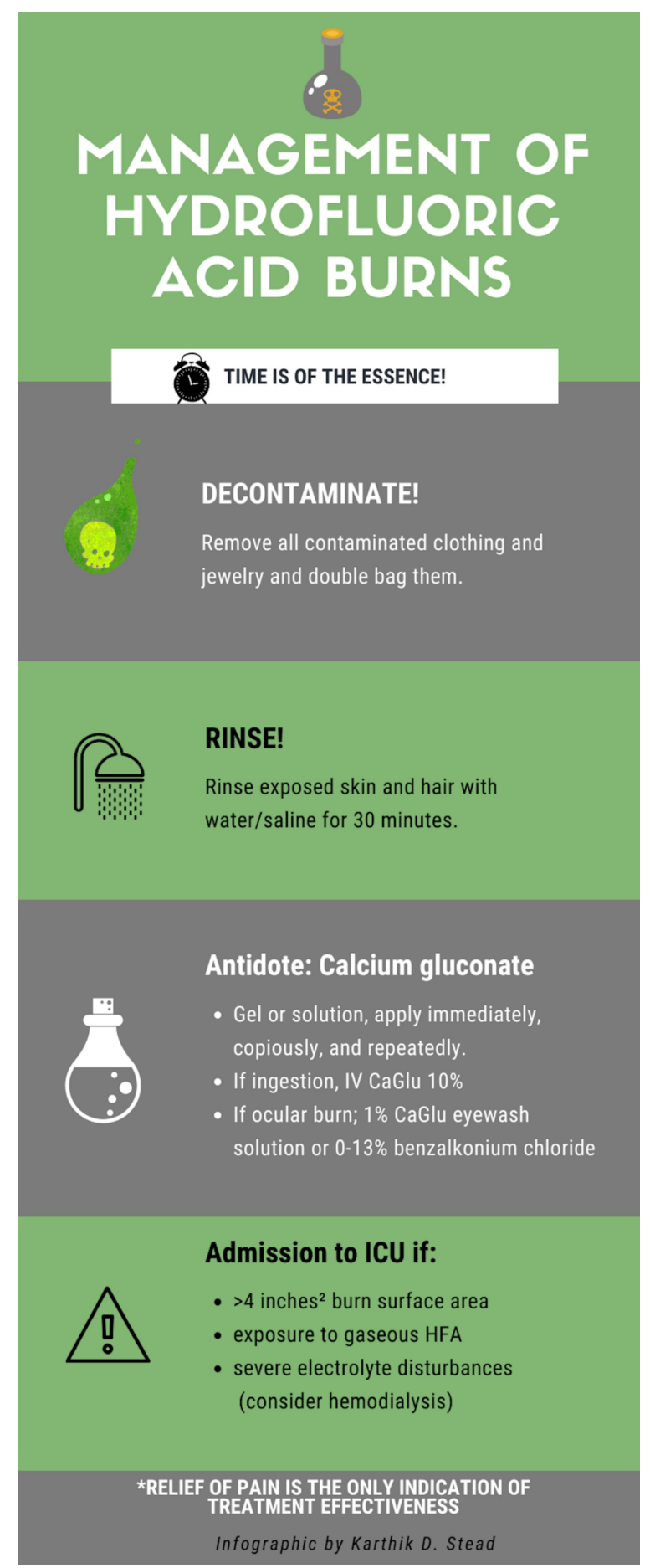

FIGURE 1: Management of hydrofluoric acid burns

The goals of treatment are as follows: (1) skin or surface decontamination; (2) neutralization of the fluoride ion; and (3) minimizing ongoing HFA absorption. Decontamination is accomplished via irrigation with copious amounts of water, saline, or soap solution ( $\mathrm{pH} 8$ or above). Ice can be applied to the to affected part to produce vasoconstriction and slowing of HFA transport into the bloodstream [8].

Neutralizing agents include CaG, benzalkonium chloride, polyethylene glycol, magnesium oxide, or Hexafluorine ${ }^{\circledR}$ (Prevor, Nesles-la-Vallée, France) [9]. CaG is the primary neutralizing agent used and comes in a variety of formulations (solution, gel, or ointment) $[2,8]$. A $10 \%$ CaG solution or $0.13 \%$ benzalkonium chloride can be used for immersion of body parts (excluding the face) or for soaking compresses. In gel form, 2.5\% CaG gel is rubbed into affected area for 15-30 minutes using HFA-resistant gloves. Calcium is used to bind to free fluoride ions, forming insoluble salts. Once $\mathrm{CaF}$ salt forms, the gel turns white. It should be wiped off and re-applied multiple times to mitigate the damage from fluoride ions [2]. Hexafluorine is a 
proprietary solution developed specifically for HFA burns. It simultaneously absorbs acid ions $(\mathrm{H}+)$ and fluoride ions (F-). Its chelation power is approximately 100 times that of $\mathrm{CaG}$ [9].

Pain is an important finding - lack of pain is the endpoint for treatment. As such, analgesic agents should be used with caution [7]. Generally acetaminophen and non-steroidal analgesics are reasonable. Throughout treatment, the patient's electrolytes need to be monitored. The healthcare team needs to monitor the patient's electrolytes, and correct hyperkalemia, hypocalcemia, and hypomagnesemia as clinically indicated.

\section{Conclusions}

HFA burns are the not most common form of burn injury seen in the ED; however, their unique properties can result in multiple forms of clinical injury. Our patient fortunately did not experience any systemic toxicity secondary to his chronic exposure. However, it is imperative that emergency personnel are aware of the acute management to optimize outcomes.

\section{Additional Information}

\section{Disclosures}

Human subjects: Consent was obtained by all participants in this study. HCA Centralized Algorithms for Research Rules on IRB Exemptions (CARRIE)/ IRB manager issued approval 2019-554. Based on the information provided and attested as true, the research plan described does not require IRB oversight. This is because you are either a) not engaging in research with human subjects as defined by federal regulations; $b$ ) engaging in research with human subjects deemed excluded from IRB oversight per 45CFR46.102(l) OR c) engaging in research with sufficient human subject protections in the design to meet one or more IRB exemption criteria set forth in 45CFR46.104. . Conflicts of interest: In compliance with the ICMJE uniform disclosure form, all authors declare the following: Payment/services info: All authors have declared that no financial support was received from any organization for the submitted work. Financial relationships: All authors have declared that they have no financial relationships at present or within the previous three years with any organizations that might have an interest in the submitted work. Other relationships: All authors have declared that there are no other relationships or activities that could appear to have influenced the submitted work.

\section{Acknowledgements}

The authors thank Karthik D. Stead for the infographic design. This research was supported (in whole or in part) by HCA Healthcare and/or an HCA Healthcare affiliated entity. The views expressed in this publication represent those of the author(s) and do not necessarily represent the official views of HCA Healthcare or any of its affiliated entities.

\section{References}

1. McKee D, Thoma A, Bailey K, Fish J: A review of hydrofluoric acid burn management. Plast Surg. 2014, 22:95-98.

2. Bajraktarova-Valjakova E, Korunoska-Stevkovska V, Georgieva S, et al.: Hydrofluoric acid: burns and systemic toxicity, protective measures, immediate and hospital medical treatment. Open Access Maced J Med Sci. 2018, 6:2257-2269. 10.3889/oamjms.2018.429

3. Hydrogen fluoride. (2019). Accessed: February 23, 2020: http://www.chemicalsafetyfacts.org/hydrogenfluoride.

4. Han HH, Kwon BY, Jung SN, Moon S-H: Importance of initial management and surgical treatment after hydrofluoric acid burn of the finger. Burns. 2017, 43:e1-e6. 10.1016/j.burns.2016.07.031

5. Schwerin DL, Hatcher JD: Hydrofluoric Acid Burns . StatPearls Publishing, Treasure Island, FL; 2019.

6. Bertolini JC: Hydrofluoric acid: a review of toxicity . J Emerg Med. 1992, 10:163-168.

7. Kirkpatrick J, Enion D, Burd D: Hydrofluoric acid burns: a review. Burns. 1995, 21:483-493. 10.1016/03054179(95)93254-h

8. Martini MC. Upfal M, Doyle C: Medical management of hydrofluoric acid exposure. J Occup Med. 1990, 32:726-731.

9. Dennerlein K, Hahn T, Göen T, Drexler H, Kilo S: Hydrofluoric acid: effects of skin decontamination on the bioavailability of fluoride. Occup Environ Med. 2018, 75:A408-A409. 10.1136/oemed-2018ICOHabstracts. 1167 